\title{
Characteristics, Comorbidities, and Data Gaps for Coronavirus Disease Deaths, Tennessee, USA
}

\author{
John James Parker, Rany Octaria, Miranda D. Smith, Samantha J. Chao, \\ Mary Beth Davis, Celia Goodson, Jon Warkentin, Denise Werner, Mary-Margaret A. Fill
}

As of March 2021, coronavirus disease (COVID-19) had led to $>500,000$ deaths in the United States, and the state of Tennessee had the fifth highest number of cases per capita. We reviewed the Tennessee Department of Health COVID-19 surveillance and chart-abstraction data during March 15-August 15, 2020. Patients who died from COVID-19 were more likely to be older, male, and Black and to have underlying conditions (hereafter comorbidities) than case-patients who survived. We found $30.4 \%$ of surviving case-patients and $20.3 \%$ of deceased patients had no comorbidity information recorded. Chart-abstraction captured a higher proportion of deceased case-patients with $\geq 1$ comorbidity $(96.3 \%)$ compared with standard surveillance deaths $(79.0 \%)$. Chart-abstraction detected higher rates of each comorbidity except for diabetes, which had similar rates among standard surveillance and chart-abstraction. Investing in public health data collection infrastructure will be beneficial for the COVID-19 pandemic and future disease outbreaks.

\footnotetext{
As of March 5, 2021, the total of deaths from coroAnavirus disease (COVID-19) reached 2,564,560 worldwide, 515,013 in the United States (1), and 11,534 in Tennessee (2). Tennessee has been particularly affected by the pandemic; as of March 5, 2021, this state had the fifth highest number of cases per 100,000 residents in the United States (3). The mortality rate for COVID-19 infection varies greatly based on patient characteristics $(4,5)$. Age and preexisting health conditions (hereafter comorbidities) have been associated with increased risk for death from COVID-19 (5-7). Cardiovascular disease (CVD),

Author affiliations: Tennessee Department of Health, Nashville, Tennessee, USA (J.J. Parker, R. Octaria, M.D. Smith, S.J. Chao, M.B. Davis, C. Goodson, J. Warkentin, D. Werner, M.-M.A. Fill); Vanderbilt University Medical Center, Nashville (J.J. Parker)

DOI: https://doi.org/10.3201/eid2710.211070
}

hypertension, diabetes, respiratory disease, cancer, kidney disease, and obesity have been associated with death; however, the strength of this association has differed among studies $(5,7)$. Although worldwide racial and ethnic minorities account for a higher proportion of COVID-19 deaths, the independent impact of race and ethnicity is unclear (8).

Challenges with data collection and reporting have made it difficult to delineate some characteristics of COVID-19 deaths. According to an assessment of surveillance data reported to the Centers for Disease Control and Prevention (CDC), 58.9\% of patients had missing comorbidity information (6). Because public health agencies gather their surveillance information from local laboratories and healthcare facilities; the completeness of their data are contingent on the local agencies obtaining and transmitting the information $(9,10)$. Consequently, mortality rate studies often focus on medical record reviews from single institutions and urban centers (11-13).

To better distinguish the characteristics of COVID-19 deaths, during March 15, 2020-May 19, 2020, the Tennessee Department of Health (TDH) implemented a supplemental chart-abstraction process for COVID-19 deaths in Tennessee. This study reviews TDH COVID-19 surveillance data and the supplemental chart review data to describe the characteristics of COVID-19 deaths in Tennessee. In addition, this study evaluates the value of a supplemental chart review process during disease outbreak surveillance.

\section{Methods}

Our study describes TDH public health data that was collected as part of COVID-19 surveillance. Definitions and protocols in place were defined by the TDH, who used CDC guidelines for their definitions of confirmed cases, probable cases, and COVID-19 deaths (14). Confirmed case-patients in Tennessee were defined as persons who had SARS-CoV-2 detected by 
using real-time reverse transcription PCR. Probable case-patients were persons who had a positive antigen test result for a respiratory specimen or persons who had no positive PCR result but met the vital records criteria or clinical criteria and had close contact to a COVID-19 case-patient during the 14 days before illness onset (15). COVID-19 deaths were defined as case-patients whose death certificate lists COVID-19 or SARS-CoV-2 as an underlying cause of death or a major condition contributing to death (16).

The sample included confirmed and probable cases in Tennessee residents who had COVID-19. We conducted investigations during March 15, 2020-August 15, 2020. Data analysis began on September 15; we used a minimum 4-week lag time to best ensure that case-patients were categorized as alive or deceased. Our primary objective was to evaluate the baseline characteristics and comorbidities of persons who died from COVID-19 in Tennessee. A secondary objective was to compare the type and quantity of data obtained through standard disease surveillance and a supplemental chart review process. The TDH Institutional Review Board (TDH-IRB\# 2020-0251) approved this study as minimal risk and waived the need for individualized consent.

\section{Data Collection}

As part of routine data entry for all COVID-19 cases, trained TDH employees completed the Human Infection with 2019 Novel Coronavirus Case Report Form (CRF) (https://www.cdc.gov/coronavirus/2019ncov/downloads/pui-form.pdf) (17) and entered the information into the National Electronic Disease Surveillance System Base System (NBS; https://www. cdc.gov/nbs/index.html). Information gathered included patient characteristics, symptoms, comorbidities, and clinical course. (The terms preexisting condition and comorbidity were used by TDH to indicate medical conditions that were present before COVID-19 infection; these terms are used interchangeably in this article.) Data collected through the CRF was the TDH standard COVID-19 disease surveillance.

In addition, during the first few months of the pandemic, the TDH created a supplemental chart review process to better classify the comorbidities and characteristics of deceased patients. This chart-abstraction project began with a group of public health professionals creating a list of 20 comorbidities to supplement the information in the standard CRF (Appendix 1, https://wwwnc.cdc.gov/EID/article/27/10/211070-App1.pdf). The chart review process creates line items for additional comorbidities. However, the CRF has 2 free text items for other chronic diseases and other underlying conditions. Therefore, we believe there is value in comparing comorbidity frequencies between the data collection groups.

Next, we added the additional chart-abstraction comorbidities to NBS to enable data entry. We then requested the medical records of all COVID-19 patients who died before May 19, 2020; 5 physicians and 1 family medicine nurse practitioner reviewed the available medical records. The provider group only reviewed complete records that included at least a complete history and physical or complete death summary. This provider group abstracted the information from the charts and added comorbidities found in the medical records to the NBS database. If there was no mention of a comorbidity, we assumed that the person did not have an underlying condition. However, when charts had gaps in documentation, the medical providers included comorbidities if there was clear evidence that the patient had a condition. For example, if a patient's chart had minimal medical history documented but had chronic problems listed in a note's plans, those problems were recorded as preexisting conditions. The group met and decided on definitions of diseases, and if there was any question on how a disease should be categorized, the individual provider would consult the group. For haste of getting this information to public health leadership, the provider's chart-abstraction work was not reviewed by a second party. After completing their review, the providers updated the information from the CRF and added additional comorbidity data into NBS. Data from the supplemental chart review project were labeled as chart-abstracted. Preliminary data from the chart review project were presented to TDH leadership at the end of May 2020.

\section{Data Characterization and Analysis}

We grouped COVID-19 case-patients into 3 groups: alive (living) case-patients, standard surveillance COVID-19 deaths, and chart-abstracted COVID-19 deaths (Figure 1). All COVID-19 cases ( $n=130,040$ ) during the study period were included in demographic analysis (Table 1). The comorbidity analysis (Tables 2, 3; Appendix 2, https://wwwnc.cdc.gov/EID/ article/27/10/21-1070-App2.pdf) excluded case-patients who had no comorbidity information recorded by only selecting cases with $\geq 1$ answers completed in the comorbidity or preexisting condition sections ( $\mathrm{n}$ $=89,270)$. In both the standard surveillance and the chart-abstraction process, if comorbidity data in the CRF was partially completed, blank items were listed as not having that condition. For race/ethnicity, we defined White as White race, non-Hispanic ethnicity, 


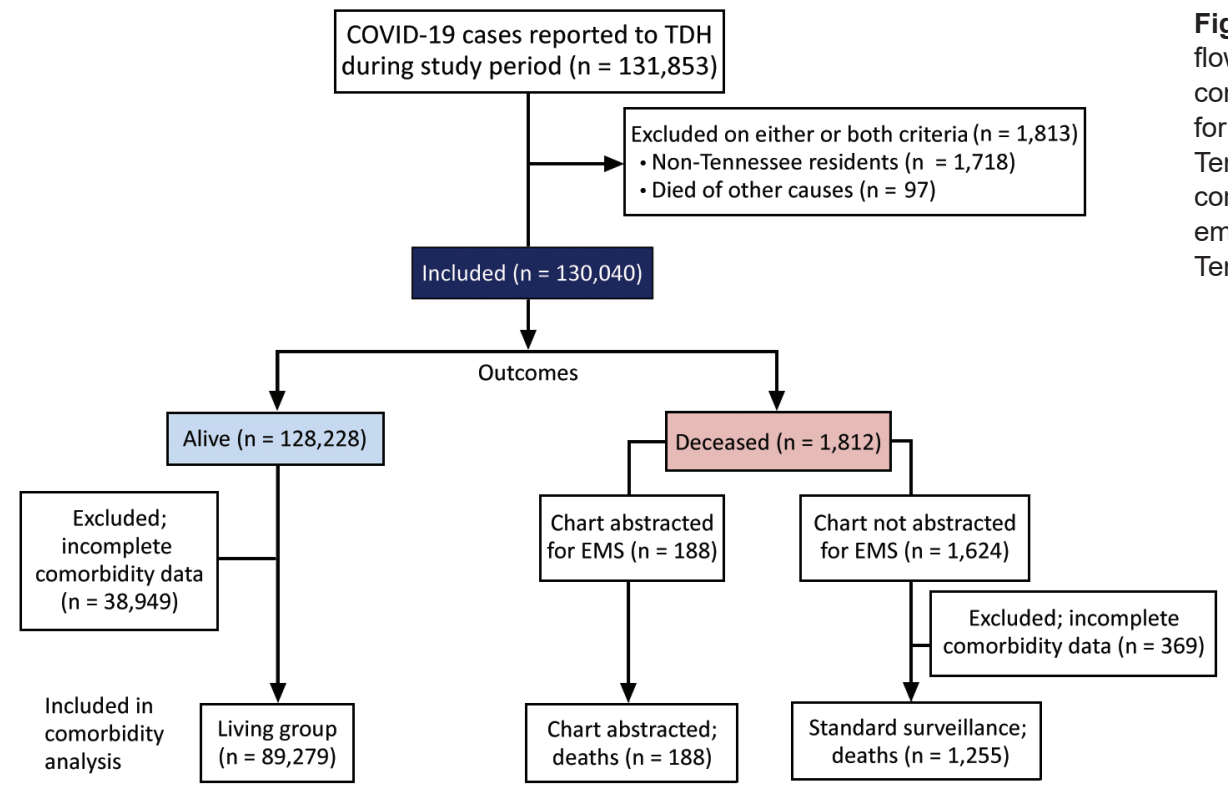

Figure 1. Data categorization flow diagram for characteristics, comorbidities, and data gaps for coronavirus disease deaths, Tennessee, USA. COVID-19, coronavirus disease; EMS, emergency medical services; TDH, Tennessee Department of Health. and Black as Black race, non-Hispanic ethnicity. We defined Hispanic as all races that selected Hispanic ethnicity. For our race/ethnicity comorbidity analysis (Tables 2, 3; Appendix 2), we excluded all other races because there were only 6 case-patients in the chartabstracted group who were not identified as White, Black, or Hispanic.

We converted comorbidities from CRFs and the chart-abstraction protocol into dichotomous variables for each condition. For the chart-abstracted deaths, we provided definitions of CVD and chronic lung disease (CLD) (Table 3) (18-20); for standard surveillance of COVID-19 deaths, we selected preexisting conditions, including CVD and CLD, on the basis of self-reports. Obesity was not included in the comorbidity analysis because the body mass index cutoffs differed between $\mathrm{CRF}$ and the chart-abstraction process. We calculated days to hospitalization by determining the difference in days between illness onset date and hospitalization admission date for patients who were hospitalized; we counted negative values (i.e., tested positive after hospitalization) as 0 and excluded probable cases from this calculation. We calculated days to specimen collection by determining the difference between the first specimen collection date for the PCR that had a positive result for SARS-CoV-2 and the illness onset date among for confirmed case-patients; probable case-patients were excluded from this calculation.

We report patient characteristics as frequencies and proportions for categorical variables and median and interquartile range for continuous variables. We compared characteristics between groups by using $\chi^{2}$ or Fisher exact tests, as appropriate, for categorical variables and $t$-test for continuous variables and performed statistical analyses by using SAS version 9.4 (SAS Institute, https:/ / www.sas.com).

\section{Results}

During the study period, we identified 131,854 COVID-19 case-patients. We excluded 1,813 casepatients because of either non-Tennessee residency or death from other causes. Of the 130,040 included case-patients, 1,812 (1.4\%) died from COVID-19. Deaths of COVID-19 case-patients were more likely to be in older, male, and Black case-patients than living case-patients (Table 1 ). The prevalence of $\geq 1$ underlying condition was higher for deceased patients $(64.8 \%)$ than for living patients $(22.6 \%)$, and this trend was true for all age groups. There were 38,949 (30.4\%) living case-patients and 369 (20.3\%) deceased casepatients who did not have any comorbidity information recorded. Therefore, for the comorbidity analysis (Tables 2, 3; Appendix 2), we excluded case-patients who did not have comorbidity data (Figure 1).

We found a difference in the case-fatality rate (CFR) for COVID-19 by race and ethnicity (White $1.9 \%$, Black $2.6 \%$, and Hispanic $0.5 \%$ ) (Table 2). The mean age of living and deceased patients also differed by race; for deceased patients the average age was 75.6 years for White patients, 69.5 years for Black patients, and 61.3 years for Hispanic patients. After stratifying by age, we found that Black patients continued to have the highest CFR. However, Hispanic case-patients $>65$ years of age had a CFR similar to or 
Table 1. Characteristics of coronavirus disease patients in Tennessee, USA, March 15-August 15, 2020

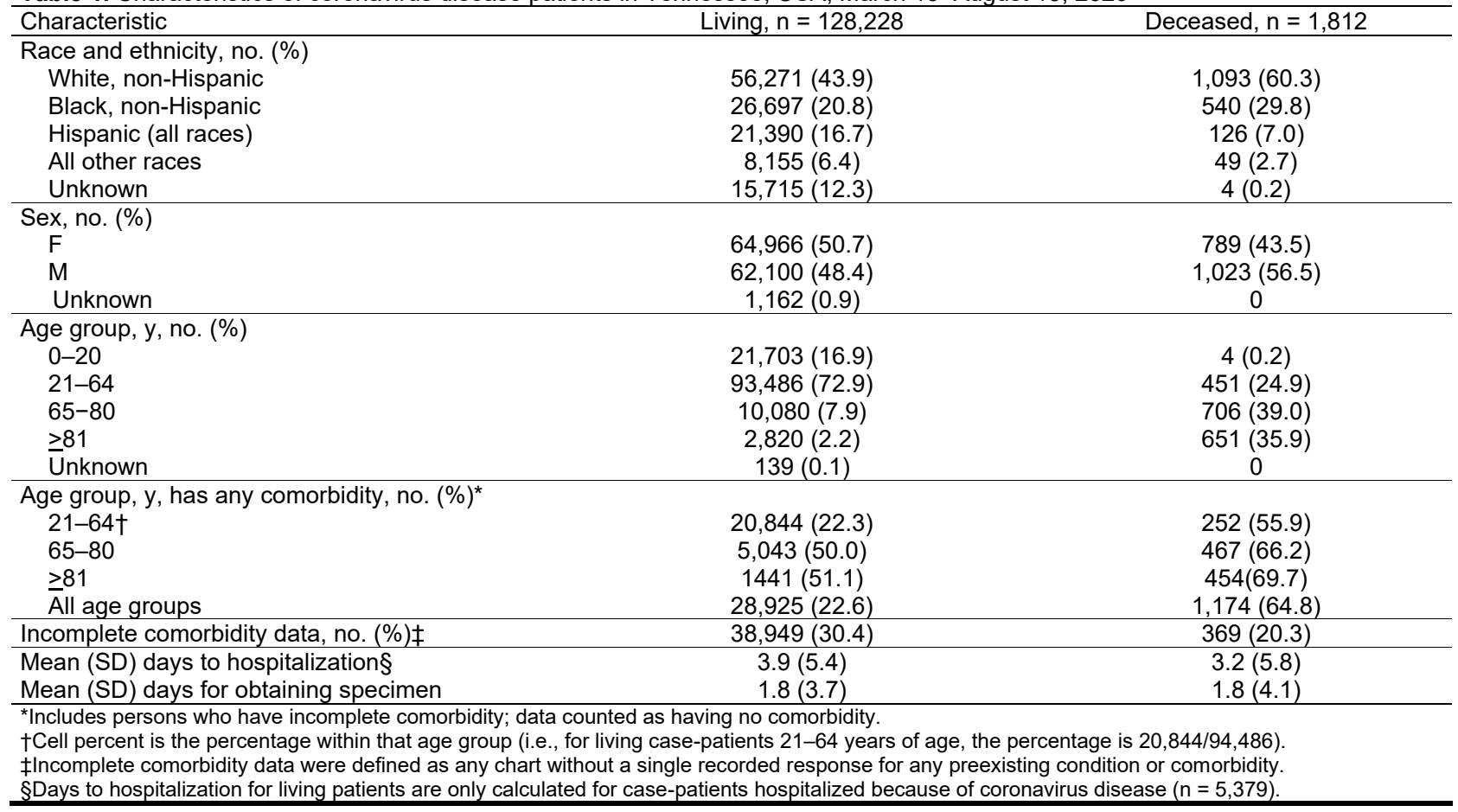

higher than that for White patients (Table 2). Hispanic patients had the lowest rate of underlying medical conditions $(64.3 \%)$ compared with White patients $(85.7 \%)$ and Black patients (91.3\%). However, Hispanic case-patients had the highest percent increase in number of comorbidities when comparing standard surveillance and chart-abstraction (Figure 2).

During March 15, 2020-May 19, 2020, there were 355 deaths; 188 of these patients who died had complete medical records available for chart abstraction. Standard surveillance and chart abstraction had comparable frequencies of race/ethnicity, proportion of females, and age (Table 3). Chart abstraction detected a higher proportion of case-patients with $>1$ comorbidity $(96.3 \%)$ compared with standard surveillance deaths $(79.0 \%)$ (Table 3$)$. After stratifying by race and age, we found that chart abstraction still found more comorbidities in each age group and race group (Figure 2; Appendix 2). Compared with standard surveillance, chart-abstracted deaths had a higher proportion of hypertension $(76.6 \%$ vs. $50.2 \%)$, CVDs $(54.3 \%$ vs.
$40.6 \%)$, CLD (29.8\% vs. $20.5 \%)$, cancer (10.6\% vs. $4.3 \%)$, and chronic renal disease (27.1\% vs. $19.0 \%)$. However, there was minimal difference in proportion of casepatients who had diabetes $(39.9 \%$ vs. $36.6 \%)$. Data for chart-abstracted deaths showed a higher proportion of current/former smoking (30.3\% vs. $15.3 \%$ ) when compared with the standard surveillance group.

\section{Discussion}

Our findings demonstrate that Tennessee has similar COVID-19 demographic trends to those that have been found throughout the United States $(21,22)$. In our sample, the average age of case-patients who died was 72.9 years compared with 38.4 years for the surviving cases. Black patients were disproportionately affected by COVID-19; at the time of our analysis, $29.8 \%$ of deaths were in Black persons, but only $17.1 \%$ of the population of Tennessee identifies as Black (23). Hispanic patients accounted for $7.0 \%$ of deaths and $5.7 \%$ of the population in Tennessee; White patients represented $60.3 \%$ of the deaths but $73.5 \%$ of the population (23).

\begin{tabular}{lccc}
\hline \multicolumn{4}{l}{ Table 2. Case-fatality rate stratified by race and age for characteristics, comorbidities, and data gaps for coronavirus disease deaths, Tennessee, USA } \\
\hline Characteristic & White, non-Hispanic, $\mathrm{n}=46,677$ & Black, non-Hispanic, $\mathrm{n}=16,669$ & Hispanic, $\mathrm{n}=17,084$ \\
\hline Mean age, y (interquartile range) & $40(25-57)$ & $37(25-52)$ & $32(21-45)$ \\
\hline Age group, y, case-fatality rate, no. $(\%)^{*}$ & $896(1.9)$ & $428(2.6)$ & $84(0.5)$ \\
$21-64 \dagger$ & $145(0.4)$ & $140(1.1)$ & $42(0.3)$ \\
$65-80$ & $344(5.9)$ & $176(12.1)$ & $31(9.0)$ \\
$\geq 81$ & $406(19.6)$ & $112(28.6)$ & $10(18.2)$ \\
\hline
\end{tabular}

*There was 1 death for a White patient ( $<1-21$ year age group) and 1 death for a Hispanic patient $(<1-21$ year age group).

†Cell percent is the case-fatality percentage for that age and race group. 
Table 3. Characteristics and comorbidities for patients with coronavirus disease, Tennessee, USA*

\begin{tabular}{|c|c|c|}
\hline \multirow[b]{2}{*}{ Characteristic } & \multicolumn{2}{|c|}{ Coronavirus disease deaths } \\
\hline & Standard surveillance, $\mathrm{n}=1,255$ & Chart-abstracted, $\mathrm{n}=188$ \\
\hline \multicolumn{3}{|l|}{ Race and ethnicity, no. (\%)† } \\
\hline White, non-Hispanic & $787(62.7)$ & $109(58.0)$ \\
\hline Black, non-Hispanic & $365(29.1)$ & $63(33.6)$ \\
\hline Hispanic (all races) & $74(5.9)$ & $10(5.3)$ \\
\hline \multicolumn{3}{|l|}{ Sex, no. (\%) } \\
\hline $\mathrm{F}$ & $549(43.7)$ & $79(42.0)$ \\
\hline $\mathrm{M}$ & $706(56.3)$ & $109(58.0)$ \\
\hline Mean age, $\mathrm{y},(\mathrm{SD})$ & $73.6(13.9)$ & $72.7(14.4)$ \\
\hline Current/former smoker, no. (\%) & $192(15.3)$ & $57(30.3)$ \\
\hline Mean no. (SD) comorbidities & $2.0(1.5)$ & $2.9(1.6)$ \\
\hline \multicolumn{3}{|c|}{ Age group, y, has any comorbidity, no. (\%)† } \\
\hline $21-64:$ Nss $=284 ; \mathrm{Nca}=50$ & $205(72.2)$ & $47(94.0)$ \\
\hline $65-80:$ Nss $=505 ; \mathrm{Nca}=63$ & $404(80)$ & $62(98.4)$ \\
\hline$\geq 81:$ Nss $=465 ; \mathrm{Nca}=74$ & $382(82.2)$ & $72(97.3)$ \\
\hline All ages: Nss $=1,254 ; \mathrm{Nca}=188$ & $991(79.0)$ & $181(96.3)$ \\
\hline \multicolumn{3}{|l|}{ Comorbidities, no. (\%) } \\
\hline Hypertension & $630(50.2)$ & $144(76.6)$ \\
\hline Cardiovascular disease $\ddagger$ & $509(40.6)$ & $102(54.3)$ \\
\hline Chronic lung disease $\S$ & $257(20.5)$ & $56(29.8)$ \\
\hline Diabetes mellitus & $459(36.6)$ & 75 (39.9) \\
\hline Obesity & $156(12.4)$ & $42(22.3)$ \\
\hline Cancer & $54(4.3)$ & $20(10.6)$ \\
\hline Chronic renal disease & $238(19.0)$ & $51(27.1)$ \\
\hline Chronic liver disease & $34(2.7)$ & $6(2.7)$ \\
\hline Immunocompromised & $76(6.1)$ & $18(9.6)$ \\
\hline Autoimmune diseases & $21(1.7)$ & $8(4.3)$ \\
\hline HIVIAIDS & $5(0.4)$ & $1(0.5)$ \\
\hline \multicolumn{3}{|c|}{$\begin{array}{l}{ }^{*} \text { Nca, no. patients who had chart abstraction for that age group; Nss, no. patients who had standard surveillance for that age group. } \\
\text { †Cell percent is the percentage in that age group (i.e., for standard surveillance case-patients } 21-64 \text { years of age, the percentage is } 205 / 284 \text { ). } \\
\text { †Defined as coronary artery disease, cerebrovascular, peripheral artery disease, heart failure, cardiomyopathies, valvular disease, myocarditis, an } \\
\text { arrythmias. Hypertension is listed separately. } \\
\text { §Defined as asthma, chronic obstructive pulmonary disease, emphysema, pulmonary hypertension, interstitial fibrosis, or sarcoidosis. }\end{array}$} \\
\hline
\end{tabular}

We also found major differences in CFRs for race/ethnicity: Hispanic patients had the lowest CFR $(0.5 \%)$ compared with that for White patients $(1.9 \%)$ and Black patients (2.6\%). The lower CFR for Hispanic patients differs from US aggregate data, in which Hispanic patients have a 2.8 times higher rate of death than do White patients (24). In Tennessee, Hispanic patients were younger than Black and White patients, and because age is the strongest predictor of death from COVID-19 $(5,11,25)$, the age difference might explain this difference in CFR for Hispanic patients in Tennessee. After stratifying by age, we found that Hispanic case-patients had CFRs similar to those for White case-patients, but Black case-patients maintained the highest CFR. In a similar fashion, CDC data have demonstrated that Hispanic patients had the largest increase in CFR once adjusted for age (21). Studies have demonstrated that race and ethnicity are associated with COVID-19 infection and death $(8,26)$. However, several reviews of in-hospital death data have demonstrated that race/ethnicity is not an independent risk factor for death after admission to the hospital $(11,26,27)$. Taken together, these data suggest that the disproportionate burden of COVID-19 deaths among racial and ethnic minorities is second- ary to systemic health and social inequities that have limited access to chronic disease management and increased the rate of COVID infection for these populations, rather than inherent difference between races and ethnicities (28).

The trend in Tennessee for comorbidities for COVID-19 patients who died also mirrors the rest of the nation (29); $22.6 \%$ of surviving case-patients had comorbidities, compared with $64.8 \%$ of those who died. After stratifying by age, we found that a higher percentage of deceased case-patients still had an underlying condition than did living case-patients. Hypertension, CVD, CLD, cancer, chronic renal disease, diabetes, and a history of smoking were more common among deceased case-patients (Appendix 1). These correlations have been found in other studies and systemic reviews in the United States and worldwide, which have had major implications for public health messaging and vaccine allocation (29).

The chart review process detected higher numbers of comorbidities than standard surveillance. In the standard surveillance group, $79.0 \%$ had a comorbidity, compared with $96.3 \%$ in the chartabstracted group. The difference between the standard surveillance and chart-abstracted group probably 


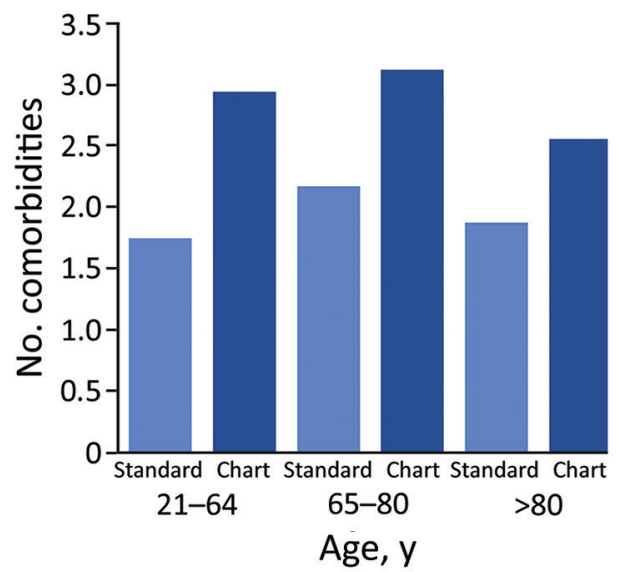

reflects issues with self-reporting and data collection. In a CDC review of COVID-19 deaths during February $12-$ May 18,2020 , a total of $58.9 \%$ of patients had missing comorbidity information according to CRFbased surveillance data (6). In our study, $30.4 \%$ of living case-patients and $20.3 \%$ of deceased case-patients had no comorbidity data recorded. The prevalence of deceased patients who had an underlying condition in our chart-abstracted group $(96.3 \%)$ is similar to that for CDC COVID-19 hospitalization records (COVIDNET), which found 405 (96.4\%) of 420 deaths had an underlying medical condition (25). This finding emphasizes that medical chart-abstraction data collects higher rates of comorbidity data than does standard public health surveillance and is a more comprehensive representation of baseline characteristics among COVID-19 patients.

For each race and age group, we found a higher number of comorbidities recorded with chart abstraction than with standard surveillance (Figure 2). In the standard surveillance group, Hispanic patients had a lower number of comorbidities than White and Black patients. Other studies have reported mixed results; Hispanic COVID-19 patients who died had more or fewer comorbidities than non-Hispanic patients $(25,27)$. In our chart-abstracted group, the total number of comorbidities for Hispanic patients was twice that of the standard surveillance group, which was the largest increase for race/ethnicity (Appendix 2). For the standard surveillance group, information was gathered by in person or telephone conversations. Therefore, language barriers and concerns about disclosure of information are 2 possible explanations for the lower number of comorbidities recorded. It has been shown that non-English-speaking patients are more likely to have inaccurate medical information, to receive lower quality care, and are at a higher risk for medical errors that result in harm $(30,31)$. Taken together, our findings demonstrate the value of chart
Figure 2. Number of comorbidities by age, race, and type of review for characteristics, comorbidities, and data gaps for coronavirus disease deaths, Tennessee, USA. abstractions to obtain accurate information for Hispanic and non-English speaking patients during disease surveillance.

We observed notable trends in the prevalence of certain comorbidities in the standard surveillance deaths compared with the chart-abstracted deaths. For example, hypertension, CVD, and CLD were detected in higher frequencies in the chart-abstracted group, and diabetes had similar rates in chart-abstraction and standard surveillance. Similarly, in multiple studies worldwide comparing self-reports and medical records, diabetes was the disease with the highest concordance (32-34); hypertension and CVD are frequently underreported in self-reports $(32,35)$. Therefore, diabetes is probably better captured by standard interview-based surveillance than other comorbidities. A meta-analysis of 87 studies determined that diabetes was the comorbidity that had the highest association with COVID-19 deaths (36). Diabetes certainly increases risk for COVID-19 deaths, but it is possible that the high accuracy of diabetes disease reporting could disproportionately increase the association between diabetes and COVID-19 death compared with other comorbidities. In addition, there is mixed evidence about whether hypertension is an independent risk factor for death (29); part of this difference could be explained by data collection and inaccurate reporting by patients.

One limitation of our study is that we used a convenience sample of COVID-19 cases collected by the TDH, which led to collection biases. For our comorbidity analysis, we excluded cases without any comorbidity information, which led to selection bias. Our chart-abstracted study occurred at the beginning of the pandemic and does not capture the burden of COVID-19 for certain ethnic and geographic groups who had more cases later in the pandemic. For example, there were 126 deaths in Hispanic COVID-19 patients and only 10 patients in the Hispanic chartabstracted group. Furthermore, our chart-abstracted 
study relied on medical charts, which created selection bias and missed patients who died outside hospitals. There might be certain groups who are more likely to have out-of-hospital deaths, but these deaths were not evaluated in our study. The data collection process was different for the standard surveillance deaths and the chart-abstracted deaths, which limited the validity of comparing the frequencies of characteristics and comorbidities. Despite these limitations, we analyzed a large number of patients, and analyzed COVID-19 demographic trends for Tennessee for comparison to other states. In addition, our chartabstraction analysis is a description of a public health study that met its goal to capture additional information compared with standard surveillance.

Our chart-based analysis showed that comorbidities related to COVID-19 deaths are more prevalent than those identified by standard public health disease surveillance. Furthermore, certain patient information tends to be reported less accurately in standard surveillance than in chart-based analysis. However, chart-based reviews are labor and time intensive, and the COVID-19 pandemic has highlighted how public health agencies are understaffed and underfunded (9). One solution for the challenges of data collection in public health disease surveillance is expansion of electronic case reporting. This type of reporting uses an interoperable, shared service infrastructure to enable automated real-time exchange of information from electronic medical records to public health agencies (37). In traditional case reporting, the most cases are reported from laboratories who lack detailed information on case demographics and often send their reports by paper copy. Therefore, public health departments receive incomplete information, which creates data gaps and distorted data, which is also apparent in our findings. In contrast, electronic case reporting provides faster and more complete data from healthcare institutions while decreasing the burden on reporters and public health departments (37). During the COVID-19 pandemic, there has been increased uptake in electronic case reporting, and continuing this trend is essential for effective disease surveillance (38).

Throughout the COVID-19 pandemic, data collected by public health agencies have been integral in identifying trends and providing information to health agencies (39). The surveillance data from Tennessee demonstrate trends in age, comorbidities, and race/ethnicity that mirror the rest of the country, and this data been used to protect those at highest risk for severe COVID-19 disease. Our study showed that chart abstraction collects more comorbidity data than standard public health disease surveillance.
In addition, certain diseases and patient groups are frequently underreported in standard surveillance, which skews public health data. These data gaps can miss at risk groups and can lead to unadvised public health action. Investment in data collection infrastructure that collects more timely and complete data will equip public health institutions, governmental organizations, and the scientific community with accurate information required to mitigate disease burden in COVID-19 and future outbreaks.

\section{Acknowledgments}

We thank members of the Division of Communicable and Environmental Disease and Emergency Preparedness, Tennessee Department of Health for their support in data collection and storage.

\section{About the Author}

Dr. Parker is an internal medicine and pediatric resident at Vanderbilt University Medical Center, Nashville, TN. His primary research interest is COVID-19.

\section{References}

1. World Health Organization. Coronavirus disease, 2020 [cited 2021 Mar 5]. https://covid19.who.int

2. Tennessee Department of Health. Data dashboard, Jan 10, 2020 [cited 2021 Mar 5]. https:/ / www.tn.gov/health/cedep/ ncov/data.html

3. Johns Hopkins Coronavirus Resource Center. All state comparison of testing efforts, Jul 6, 2021 [cited 2021 Mar 5]. https://coronavirus.jhu.edu/testing/states-comparison

4. Jin J, Agarwala N, Kundu P, Harvey B, Zhang Y, Wallace $\mathrm{E}$, et al. Individual and community-level risk for COVID-19 mortality in the United States. Nat Med. 2021;27:264-9. https://doi.org/10.1038/s41591-020-01191-8

5. Williamson EJ, Walker AJ, Bhaskaran K, Bacon S, Bates C, Morton CE, et al. Factors associated with COVID-19-related death using OpenSAFELY. Nature. 2020;584:430-6. https://doi.org/10.1038/s41586-020-2521-4

6. Wortham JM, Lee JT, Althomsons S, Latash J, Davidson A, Guerra K, et al. Characteristics of persons who died with COVID-19-United States, February 12-May 18, 2020. MMWR Morb Mortal Wkly Rep. 2020;69:923-9. https:/ / doi.org/10.15585/ mmwr.mm6928e1

7. Deng G, Yin M, Chen X, Zeng F. Clinical determinants for fatality of 44,672 patients with COVID-19. Crit Care. 2020;24:179. https://doi.org/10.1186/s13054-020-02902-w

8. Pan D, Sze S, Minhas JS, Bangash MN, Pareek N, Divall P, et al. The impact of ethnicity on clinical outcomes in COVID-19: a systematic review. EClinicalMedicine. 2020;23:100404. https://doi.org/10.1016/j.eclinm.2020.100404

9. Dixon BE, Caine VA, Halverson PK. Deficient response to COVID-19 makes the case for evolving the public health system. Am J Prev Med. 2020;59:887-91. https:/ / doi.org/ 10.1016/j.amepre.2020.07.024

10. Centers for Disease Control and Prevention. Improving public health Surveillance, 2020 [cited 2021 Jun 2]. https://www.cdc.gov/surveillance/improving-surveillance/index.html 
11. Price-Haywood EG, Burton J, Fort D, Seoane L. Hospitalization and mortality among black patients and white patients with COVID-19. N Engl J Med. 2020;382:253443. https://doi.org/10.1056/NEJMsa2011686

12. Richardson S, Hirsch JS, Narasimhan M, Crawford JM, McGinn T, Davidson KW, et al.; Northwell COVID-19 Research Consortium. Presenting characteristics, comorbidities, and outcomes among 5,700 patients hospitalized with COVID-19 in the New York City area. JAMA. 2020;323:2052-9. https:// doi.org/10.1001/ jama.2020.6775

13. Mueller JT, McConnell K, Burow PB, Pofahl K, Merdjanoff AA, Farrell J. Impacts of the COVID-19 pandemic on rural America. Proc Natl Acad Sci U S A. 2021;118:2019378118. https://doi.org/10.1073/pnas.2019378118

14. Centers for Disease Control and Prevention. Coronavirus disease 2019 (COVID-19), 2020. Interim case definition, approved August 5, 2020 [cited 2021 Feb 13]. https:/ / ndc.services.cdc.gov/case-definitions/ coronavirus-disease -2019-2020-08-05

15. Council of State and Editorial Epidemiologists. Interim20-ID-02_COVID-19. [cited 2020 Sep 8]. https:/ / cdn.ymaws. com/www.cste.org/resource/resmgr/ps/positionstatement 2020/Interim-20-ID-02_COVID-19.pdf

16. Tennessee Department of Health. COVID-19 case definition [cited 2021 Jul 6]. https://www.tn.gov/content/dam/tn/ health/documents/cedep/novel-coronavirus/COVIDCase-Definition.pdf

17. Centers for Disease Control and Prevention. pui-form [cited 2020 Jun 6]. https://www.cdc.gov/coronavirus/2019-ncov/ downloads/pui-form.pdf

18. American Heart Association. What is cardiovascular disease? [cited 2020 Dec 14]. https://www.heart.org/en/ health-topics/consumer-healthcare/what-is-cardiovasculardisease

19. World Health Organization. About cardiovascular diseases [cited 2020 Dec 14]. https:/ / www.who.int/cardiovascular_ diseases/about_cvd/en/

20. World Health Organization. Chronic respiratory diseases [cited 2020 Dec 14]. https:/ / www.who.int/westernpacific/ health-topics/chronic-respiratory-diseases

21. Centers for Disease Control and Prevention. COVID-19 provisional counts: health disparities, 2020 [cited 2020 Dec 13]. https://www.cdc.gov/nchs/nvss/vsrr/covid19/ health_disparities.htm

22. Centers for Disease Control and Prevention. COVID-19 provisional counts: weekly updates by select demographic and geographic characteristics, 2020 [cited 2020 Dec 13]. https://www.cdc.gov/nchs/nvss/vsrr/covid_weekly/ index.htm

23. US Census Bureau. Quick Facts Tennessee [cited 2020 Dec 12]. https://www.census.gov/quickfacts/TN

24. Centers for Disease Control and Prevention. COVID-19 hospitalization and death by race/ethnicity, 2020 [cited 2020 Dec 23]. https:/ / www.cdc.gov/coronavirus/2019-ncov/ covid-data/investigations-discovery/hospitalizationdeath-by-race-ethnicity.html

25. Kim L, Garg S, O'Halloran A, Whitaker M, Pham H, Anderson EJ, et al. Risk Factors for intensive care unit admission and in-hospital mortality among hospitalized adults identified through the U.S. coronavirus disease 2019 (COVID-19)-associated Hospitalization Surveillance Network (COVID-NET). Clin Infect Dis. 2021;72:e206-14. https://doi.org/10.1093/cid/ciaa1012

26. Golestaneh L, Neugarten J, Fisher M, Billett HH, Gil MR, Johns T, et al. The association of race and
COVID-19 mortality. EClinicalMedicine. 2020;25:100455. https://doi.org/10.1016/j.eclinm.2020.100455

27. xKabarriti R, Brodin NP, Maron MI, Guha C, Kalnicki S, Garg MK, et al. Association of race and ethnicity with comorbidities and survival among patients with COVID-19 at an urban medical center in New York. JAMA Netw Open. 2020;3:e2019795. https:/ / doi.org/10.1001/ jamanetworkopen.2020.19795

28. Centers for Disease Control and Prevention. Communities, schools, workplaces, and events, 2020 [cited 2020 Dec 13]. https://www.cdc.gov/coronavirus/2019-ncov/ community/health-equity/race-ethnicity.html

29. Centers for Disease Control and Prevention. COVID-19 and your health, 2020 [cited 2020 Dec 12]. https:/ / www.cdc.gov/ coronavirus/2019-ncov/need-extra-precautions / evidence-table.html

30. Divi C, Koss RG, Schmaltz SP, Loeb JM. Language proficiency and adverse events in US hospitals: a pilot study. Int J Qual Health Care. 2007;19:60-7. https:/ / doi.org/ 10.1093/intqhe/mzl069

31. Green AR, Nze C. Language-based Inequity in health care: who is the "poor historian"? AMA J Ethics. 2017;19:263-71. https:// doi.org/10.1001/journalofethics.2017.19.3.medu1-1703

32. Frost M, Wraae K, Gudex C, Nielsen T, Brixen K, Hagen C, et al. Chronic diseases in elderly men: underreporting and underdiagnosis. Age Ageing. 2012;41:177-83. https:// doi.org/10.1093/ageing/afr153

33. van den Akker M, van Steenkiste B, Krutwagen E, Metsemakers JFM. Disease or no disease? Disagreement on diagnoses between self-reports and medical records of adult patients. Eur J Gen Pract. 2015;21:45-51. https:/ / doi.org/ 10.3109/13814788.2014.907266

34. Kriegsman DM, Penninx BW, van Eijk JT, Boeke AJ, Deeg DJ. Self-reports and general practitioner information on the presence of chronic diseases in community dwelling elderly. A study on the accuracy of patients' self-reports and on determinants of inaccuracy. J Clin Epidemiol. 1996;49:140717. https:/ / doi.org/10.1016/S0895-4356(96)00274-0

35. Peterson KL, Jacobs JP, Allender S, Alston LV, Nichols M. Characterising the extent of misreporting of high blood pressure, high cholesterol, and diabetes using the Australian Health Survey. BMC Public Health. 2016;16:695. https://doi.org/10.1186/s12889-016-3389-y

36. Corona G, Pizzocaro A, Vena W, Rastrelli G, Semeraro F, Isidori AM, et al. Diabetes is most important cause for mortality in COVID-19 hospitalized patients: systematic review and meta-analysis. Rev Endocr Metab Disord. 2021;22:275-96. https:/ / doi.org/10.1007/s11154-021-09630-8

37. Centers for Disease Control and Prevention. How does electronic case reporting work? 2020 [cited 2021 Feb 15]. https://www.cdc.gov/ecr/index.html

38. American Medical Association. Why it's essential to improve data collection and reporting [cited $2021 \mathrm{Feb} 15]$. https:/ / www.ama-assn.org/delivering-care/public-health/ why-it-s-essential-improve-data-collection-and-reporting

39. Centers for Disease Control and Prevention. Coronavirus disease 2019 (COVID-19): transmission, 2020 [cited 2020 Dec 26]. https:/ / www.cdc.gov/coronavirus/2019-ncov/ covid-data/faq-surveillance.html

Address for correspondence: J.J. Parker, Attn: Mary-Margaret Fill, 710 James Robertson Pkwy, 4th Fl, Andrew Johnson Tower, Communicable and Environmental Diseases and Emergency Preparedness, Nashville, TN 37243, USA; email: jjparker847@gmail.com 